\title{
Augmenting Media Literacy with Automatic Characterization of News along Pragmatic Dimensions
}

\section{Miriam Boon}

Northwestern University

Evanston, IL 60201, USA

MiriamBoon2012@u.northwestern.

edu
Permission to make digital or hard copies of part or all of this work for personal or classroom use is granted without fee provided that copies are not made or distributed for profit or commercial advantage and that copies bear this notice and the full citation on the first page. Copyrights for third-party components of this work must be honored. For all other uses, contact the Owner/Author.

Copyright is held by the owner/author(s).

CSCW '17 Companion, February 25 - March 01, 2017, Portland, OR, USA ACM 978-1-4503-4688-7/17/02.

http://dx.doi.org/10.1145/3022198.3024948

\begin{abstract}
Media literacy allows individuals to better interpret the information they need to absorb to contribute to our democratic, knowledge-based society. I propose that by automatically notifying readers of an article's problematic pragmatic characteristics, an application could augment their media literacy. I describe two characteristics for which I have been building automatic classifiers - factiness, and tropes as narrative frames and discuss the status of each project.
\end{abstract}

\section{Author Keywords}

Computational journalism; information retrieval;

machine learning; natural language processing.

\section{ACM Classification Keywords}

H.5.m. Information interfaces and presentation (e.g. $\mathrm{HCI}$ ): Miscellaneous; H.3.m. Information storage and retrieval: Miscellaneous.

\section{Introduction}

The flow of information is essential in a democratic, knowledge-based society. The information we are able to access online in the form of articles, however, is far from perfect. Articles can be factually incorrect, incomplete, or misleading. They may also promote an 


\section{Pragmatics}

The hard, mechanical aspects

of communication - syntax

(e.g. grammar) and semantics (e.g. words used)

- are not always sufficient to interpret content, or understand how individuals have interpreted content. Pragmatics is the linguistic branch that deals with those aspects of communication that syntax and semantics cannot address.

\section{Example 1}

An article about Al Sharpton's daughter sueing the City of New York under questionable circumstances. A Twitter user, aware of Al Sharpton's history of arguably spurious lawsuits, commented, "The apple doesn't fall far from the tree." As a Canadian, I had no idea why.

\section{Example 2}

An article written in a scientific style that does not have scientific content. The scientific style would lend the article authority for many readers. agenda, attempt to persuade, or claim inappropriate authority.

If the article does one or more of these things in an obvious manner, many readers may recognize these flaws and adjust their interpretation of the article content accordingly. But when the article is subtle in its agenda, persuasion, or claims to authority, the flow of information to readers with low media literacy becomes corrupted with misinformation.

While media literacy education attempts to address this problem by teaching the relevant skills to students, my work considers how technology can augment a reader's existing media literacy. Three types of applications could accomplish this goal: (1) A news browser that filters out problematic content; (2) a system that identifies the issue with the article, and informs the reader so that they can approach it with care; (3) a system that identifies the events the article describes, and finds other articles about the same story, but with fewer flaws or contrasting characterizations, and recommends them. In particular, I believe the latter two options will promote greater media literacy and mindfulness among readers.

All three methods require the same underlying technology: automatic characterization of articles with respect to the dimensions we deem problematic. Technologically, this turns out to be particularly challenging. A great deal of work has addressed the automatic classification of news articles, but it has generally focused on article topic. But topics are very strongly related to words, making these straightforward, semantic models. The subtle flaws we wish to identify are most often pragmatic (see sidebar) in nature.

My dissertation will explore the automatic detection of several pragmatic dimensions in news articles.

Typically, each dimension I explore requires a unique data set and a different computational approach. Given space constraints, I will briefly discuss two below.

\section{Example 1: Factiness}

I define factiness as "The quality of being perceived as factual, regardless of actual truth." Thus, an article that scores high in factiness, but in fact expresses opinions or contains false information, is claiming inappropriate authority in order to deceive or persuade. From a cognitive perspective, I assume that readers have heuristics they use to assess an article's factuality without actually fact-checking it. Factiness, then, is an ensemble model consisting of those heuristics. My task is to identify, formalize, and combine those heuristics into a single predictive model.

I explore this dimension using a data set harvested from NewsTrust [4, 10]. Launched in 2005 and defunct as of 2014, NewsTrust allowed readers to rate articles from any news site by answering one or more of several specific questions [6]. I leveraged the question, "Is this story factual?" as a measure of factiness. I was able to retrieve relatively clean text for 806 articles rated based on 'factiness'.

The first heuristic I investigated was information density. Specifically, I posited that given two documents of equal length, the average reader would perceive the document with more factoids (fact-like claims) as being more factual [1]. I developed a 


\section{Research Questions}

Each dimension addresses separate research questions while driving towards the same applied goal.

Factiness

RQ1. What text-based heuristics inform a reader's assessment of factiness?

RQ2. What, if any, relationship does factiness have with factuality?

\section{Tropes}

RQ1. What proportion of news stories are characterized by tropes?

RQ2. How often are tropes identified based only on the reader's personal context, as opposed to being present in the article text as a narrative frame?

RQ3. Can a machine learning approach detect tropes with any accuracy? If not, can a computationally low-cost hybrid approach do so measure of information density and assessed its correlation with factiness as rated by NewsTrust readers. The results were promising but require further investigation.

I next posited that there might be word groups that readers use as heuristics. I used an algorithm that identifies and ranks the words that are most predictive of factiness. I found that these included a disproportionate number of words related to measurement, expressions of doubt, and expressions of certainty. Next steps will involve constructing conceptual word groups based on this insight, and analyzing their relationship with factiness.

The final step for this dimension is to discover the relationship between factiness and factuality, and determine if deceptive factiness can be identified automatically. I will acquire a corpus of news articles that have been fact-checked, and explore the relationship between factiness and factuality.

\section{Example 2: Tropes as Narrative Frames}

Our indoctrination in narrative frames begins at a very young age [2]. We each internalize our own unique mix of mythic and modern archetypes, informed by our family and culture, and they become a critical part of how we make sense of - and describe - the world around us.

Some of these tropes are more widely known than others. "David and Goliath," "Achilles' Heel," and "The Emperor's New Clothes" are just a few of the bestknown examples. The narrative genres in which these tropes are most obviously visible - and from which they often derive - are fables, folk tales, epigrams, and even jokes, and people are often spontaneously reminded of these kinds of narratives by stories and situations that potentially embody them. Tropes are short forms for very abstract, complex, and highlystructured concepts.

When used as narrative frames for news articles, they can both clarify and entertain. But when misused, they can mislead and introduce bias that can be difficult for a reader to recognize $[5,7-9]$. For example, by framing a story to match a trope with a villain or hero, the author can dispose readers towards positive or negative impressions of real people or organizations without ever explicitly criticizing them.

The News Tropes project has two phases. The first phase involves the use of implicit crowdsourcing to find examples of stories that are perceived as having a particular theme. Version 0.1 of this system finds tweets that include a variation on one of our trope names along with a link to a news story. A trial run searching for 20 tropes over the course of 16 days returned 3513 trope-relevant tweets pertaining to 1414 articles. We found that the system yielded appropriate labels for a wide variety of articles. Having proved the concept, we proceeded to develop Version 0.2, which will search for more than 100 themes, and features numerous improvements to the algorithm.

The second phase of the project, currently underway, crowdsources article labels using the system from Phase 1 . We will then attempt to train a machine learning algorithm to predict those labels based on the article text. Typical approaches to automatic identification of themes, tropes, and metaphors based on text use computationally expensive methods [3]. A 


\section{Goals for the CSCW Doctoral Colloquium}

I am excited about getting feedback on my work from members of the CSCW community. I believe the participants and faculty panelists will have fresh perspectives that will be of immediate value for me at this stage of my work. But I also view this intensive event as an incredible opportunity to rapidly absorb and assimilate CSCW community norms and values. And that has enduring value, particularly as I have not yet had the opportunity to attend CSCW.

I have an unusual and varied background. Thus, it is my hope that I will be able to return value for value, and provide a unique perspective for other participants. successful machine learning model would potentially be far more scalable and robust.

\section{Acknowledgements}

Many thanks to Larry Birnbaum, Darren Gergle, and Jason Cohn. This work was supported by the Technology and Social Behavior program at Northwestern University, the National Science Foundation (grant numbers IIS-0856058 and IIS0917261/001), the Knight Foundation, and Google.

\section{References}

[1] Boon, M. 2014. Information density, Heaps' Law, and perception of factiness in news. Proceedings of the ACL 2014 Workshop on Language Technologies and Computational Social Science. (2014), 33-37.

[2] Ettema, J. 2004. Crafting Cultural Resonance: Imaginative Power in Daily Journalism. Conference Papers -- International Communication Association. (Annual Meeting 2004), 1.

[3] Finlayson, M.A. 2011. The Story Workbench: An Extensible Semi-Automatic Text Annotation Tool. Intelligent Narrative Technologies (2011).

\section{[4] Florin, F. 2009. NewsTrust Communications} 2009 Report.

[5] Hervás, R. and Finlayson, M.A. 2010. The prevalence of descriptive referring expressions in news and narrative. Proceedings of the ACL 2010 Conference Short Papers (2010), 49-54.
[6] Lampe, C. and Garrett, R.K. 2007. It's All News to Me: The Effect of Instruments on Ratings Provision. System Sciences, 2007. HICSS 2007. 40th Annual Hawaii International Conference on (2007), 180b180b.

[7] Matthes, J. 2009. What's in a frame? A content analysis of media framing studies in the world's leading communication journals, 1990-2005. Journalism \& Mass Communication Quarterly. 86, 2 (2009), 349-367.

[8] Miller, M.M. 1997. Frame mapping and analysis of news coverage of contentious issues. Social Science Computer Review. 15, 4 (1997), 367-378.

[9] Pan, Z. and Kosicki, G.M. 1993. Framing analysis: An approach to news discourse. Political Communication. 10, 1 (1993), 55-75.

[10] Survey Report Summary - NewsTrust: 2006. http://www.newstrust.net/survey/report. Accessed: 2013-03-21. 